\title{
High-speed 2-D and 3-D animation on the IBM PC/XT/AT
}

\author{
JACK M. LOOMIS and DAVID W. EBY \\ University of California, Santa Barbara, California
}

\begin{abstract}
This article describes programs for performing high-speed two-dimensional (2-D) and threedimensional (3-D) animation on IBM PC/XT/AT and compatible machines that use the color/graphics adapter. Machine language routines interfaced through BASIC allow the user to store screen images in memory as they are created by user software and then to display the sequence of images at rates of up to 60 frames per second. A BASICA 3-D graphics editor that can be used with the routines permits the user to create wire-frame objects and to move them about rigidly in conceptual space while projecting them onto the display screen. These programs should find application in the study of visual perception and cognition.
\end{abstract}

Although the computer saves much time, effort, and expense in experimental research, its greatest value is in enabling research that would otherwise be impossible. In the study of visual perception, for example, much of the research envisioned by Gibson $(1950,1979)$ involving higher order optical variables was until recently impossible to carry out properly, since some stimulus variables (e.g., texture element size) are invariably coupled with others (e.g., optical motion of objects moving in depth) in imagery produced by conventional mechanical and optical means, such as the shadowcaster. The computer, however, allows one to create visual imagery of vast complexity while decoupling those stimulus variables that covary in the real world. Thus the computer has truly opened up the experimental investigation of the perception of three-dimensional structure from optical motion (Badler \& Tsotsos, 1986; Braunstein, 1976; Todd, 1985; Ullman, 1979). In the world of microcomputers, however, 8-bit machines have been of limited use to the researcher interested in research-level animation because of deficiencies in speed and memory. The more powerful 16- and 32-bit machines, in contrast, are proving quite adequate for performing interesting research requiring high-speed animation. This article presents machine language routines interfaced through BASIC for performing animation on the IBM PC/XT/AT and compatible machines that use the color/graphics adapter. These routines should prove useful in studies of visual perception and cognition for which real-time generation of imagery is too slow for the desired application. In addition, the article describes a BASIC editor for creating and moving three-dimensional (3-D) objects while projecting them onto the video screen. This editor, or one like it, when used in conjunction with the animation routines, should find application in the study

The authors are grateful to Richard Parker and Larry Murdock for programming assistance and to the anonymous reviewers for helpfu comments on the manuscript. Address correspondence to Jack $M$ Loomis, Department of Psychology, Univessity of California, Santa Barbara, CA 93106. of both motion perception and the perception of structure from motion.

\section{ANIMATION ROUTINES}

The two basic approaches to performing animation are: (1) computing and displaying the image sequence in real time, and (2) computing and storing each image of the sequence in advance and then, during display, moving each image into video memory in rapid succession. The former approach is more flexible and permits adaptive control of the imagery by the user, such as in flight simulation; however, stand-alone 16-bit machines are not sufficiently powerful to do real-time computation of complex imagery at high frame rates. By performing the computation in advance, the second approach permits one to do complex 2-D and 3-D animation at frame rates of up to 60 per second. However, in avoiding the necessity for real-time computation, one compensates with the need for large amounts of internal and external memory. Because of our desire for speed, we have chosen the second approach.

To obtain the desired speed, the three routines used in this work, CLRMEM, STORE, and DISPLAY, were written in assembly language; they are the three procedures of ANIMATE.ASM given in Listing 1. These routines can be used by anyone with minimal knowledge of BASIC using the interface program ANIMATE.BAS, given in Listing 2. Because this BASIC program stores the machine language routines of ANIMATE.ASM within the DATA statements and pokes them into memory (in line 30), it functions as a stand-alone program.

\section{Program Description}

A brief description of the machine language routines and of the BASIC interface program is presented in this section. As part of the initialization section (lines 10-110), the program calls the CLRMEM routine, which clears the portion of random access memory (RAM) that will be 
used to store the images in the animation sequence. The second section of the program (lines 120-190 and the subroutine beginning at line 2000) creates the image of each frame using the graphics commands of BASIC and then calls the STORE routine, which stores each image in successive portions of RAM. It does this in the following way. Starting at the top of display memory (address $=\mathrm{B} 8000 \mathrm{H}$ ), the routine scans for nonzero bytes, which correspond to illuminated portions of the screen. Each time a nonzero byte is encountered, its value and address are stored in successive locations of free RAM. After all images in the sequence have been stored, the BASIC program proceeds to the display section (lines 200 280). The DISPLAY routine starts at the beginning of the image sequence and moves the stored bytes of each stored image into display memory, causing the corresponding part of the video screen to be illuminated. Prior to displaying each image, the previous screen image is erased. The beginning of erasure is synchronized to the vertical retrace pulse (Davies, 1985) so that graphical output of the computer remains in phase with the raster scan of the video monitor. The number of video scans during which each image appears on the video screen is controlled by the parameter NUMREP. A value of 1 results in animation rates of 60 frames per second.

\section{Using the Programs}

Use of the BASIC interface program is quite simple. After entering the program into memory, one can alter the graphics subroutine beginning at line 2000 to create whatever image is desired for each frame of the sequence. In the present example, a single rectangle is drawn and stepped across the screen in successive frames. The example also uses medium-resolution graphics (SCREEN 1), but high-resolution graphics (SCREEN 2) may be used instead. In addition, text may be mixed with graphics within each image. If color is desired, either palette of medium-resolution graphics can be used, although an RGB monitor may be required for good results.

The program and subroutines of Listing 2 were developed and tested on an IBM PC with 512K of RAM. They should run without modification on any normally configured IBM PC/XT/AT with at least this amount of memory. For computers with less memory, the value in the DEF SEG statement in line 20 must be reduced; users of computers with more memory may wish to increase the value. This value specifies the starting address of free RAM at which the machine language routines are stored; it is near the upper limit of expansion memory. Consult the IBM BASIC manual or other texts (e.g., Davies, 1985) for information on the DEF SEG statement.

Image sequences are stored in free RAM above the BASIC interpreter and its workspace but below the locations of the machine language routines. IBM PC/XT/AT computers treat memory in $64 \mathrm{~K}$ segments. For the present purposes, we will consider nonoverlapping $64 \mathrm{~K}$ segments and number them beginning at low memory with 0 . The machine on which these programs were developed had 8 segments of available RAM $(8 \times 64 \mathrm{~K}=512 \mathrm{~K})$; accordingly, the segments were numbered from 0 to 7 . Because the basic interpreter and its workspace normally reside within segments 0 and 1 and because the machine language routines are poked into segment 7 by the program, segments 2-6 (or 320K of memory) are available for storing image sequences. A user having a computer with more memory may wish to set the segment register in line 20 to a higher value and thus have more RAM available for storing image sequences. Each $64 \mathrm{~K}$ segment can hold 85 frames of 256 bytes each or the equivalent (e.g., 170 frames of 128 bytes). If the user's system is configured with BASIC and its workspace located within segments other than 0 and 1 , the user may experience difficulty when storing image sequences in the segments suggested; memory conflicts can be avoided by storing sequences above the BASIC workspace (see either Davies, 1985, or Rollins, 1985).

When the program is run, it first requests the number of the starting segment. In the present example, the value 2 would be an appropriate response; if memory conflicts are experienced, the value 3 ought to be tried. At this point, the program requests the number of bytes expected for each image and then the number of frames in the sequence. As a general rule, the number of bytes per image should correspond to the number of illuminated pixels on the screen (whether graphics or text). However, since each byte of display memory represents 4 horizontally adjacent pixels in medium-resolution graphics, one can store more pixels for the same number of bytes when the images are compact. If the number of bytes in an image exceeds the number entered, the excess bytes will be overwritten by the subsequent frame. Before image creation and storage begins, the program calls the CLRMEM routine to clear RAM for storage. The total number of bytes and the number of segments cleared are computed by the expressions in line 80; these are labeled NUMBYTES and NUMBER, respectively. Care must be taken not to specify a sequence of images that exceeds the amount of available storage, for clearing either the BASIC program or the machine language routines will cause the system to crash.

After clearing RAM, the program creates and stores the image sequence. When completed, the program requests the number of raster scans per frame and the number of times through the sequence. A value of up to 65535 may be entered for either. Because there are 60 raster scans per second, the minimum duration per frame (corresponding to a single scan) is $16.7 \mathrm{msec}$.

If the user wishes to use a compiled BASIC program to speed creation of each image within the sequence, CLRMEM and STORE can be assembled and then linked with the image-generation program (see Rollins, 1985, for a discussion of interfacing to other high-level languages). We have done this with considerable time sav- 
ings when complex images are being computed; however, we have also experienced some loss in available RAM for storing the image sequence because of memory conflicts.

The user may save image sequences to disk using the BSAVE statement of BASIC. To do so, the user must first set the code segment register, which determines the $64 \mathrm{~K}$ segment to be used. This is accomplished by executing a BASIC statement of the form DEF SEG $=4096 * S N$, where $\mathrm{SN}$ is the number used in the interface program to refer to the starting segment. Thus if the sequence were stored beginning at segment 2 , the statement to be executed would be DEF SEG $=8192$. If the image sequence created were named SEQUENCE and occupied less than one $64 \mathrm{~K}$ segment of RAM, one could then save it to disk with the statement BSAVE SEQUENCE,0,N, where $N$ is the value of NUMBYTES in line 80 of the interface program. Reloading the sequence later from disk could be accomplished by the statement BLOAD SEQUENCE,0. Sequences occupying more than one $64 \mathrm{~K}$ segment of RAM must have each of their segments saved, each time changing the segment value in the DEF SEG statement.

Finally, image sequences can be taped by taking the composite video output of the color/graphics adapter as input to a high-bandwidth videocassette recorder. Because the image sequence is already synchronized to the video signal, no special interface is required.

\section{Performance}

Animation rates are possible up to the video scan rate of 60 rasters per second. At this rate, an IBM PC running at $4.77 \mathrm{MHz}$ can transfer images of roughly 250 bytes per image. Because erasure of the previous screen image and transfer of the next image proceed from top to bottom in advance of the video scan but at a slower rate, the user can achieve transfer of more complex images simply by locating the top of each image some distance down from the top of the graphics screen. The size limit of 250 bytes per image requires that the image be confined to the lower 160 rows (out of 200) of the graphics screen, although this limit depends somewhat upon image configuration. A PC AT running at $6 \mathrm{MHz}$ can transfer 250 bytes without restriction as to location and over 500 bytes per image when each image is confined to the lower 170 rows. If 2,3 , or more raster scans per image are selected, image complexity in bytes can be much greater, although some pixels may be displayed one raster scan less than others (resulting in some nonuniformity of brightness from top to bottom). One solution for maximizing image complexity without sacrificing a high frame rate is to rewrite the DISPLAY routine for a graphics board having two display buffers and then to load one buffer with an image while displaying the other. With this double-buffering technique, one can transfer images of at least 500 bytes each at a $60-\mathrm{Hz}$ frame rate on an
IBM PC and 1000 bytes each on a PC AT. However, since each 1000 -byte image takes up 3000 bytes of RAM ( 2 bytes for the screen address and 1 for its content), the internal and external storage demands of complex image sequences and the time to load them from external memory take on major importance. Incidentally, because of the way in which images are stored in RAM, using a different graphics board with higher spatial resolution than the IBM color/graphics adapter makes no additional storage demands, provided that the display buffer does not exceed $64 \mathrm{~K}$.

\section{3-D GRAPHICS EDITOR}

This section of the paper provides a description of a 3-D graphics editor (in BASICA) that makes use of the animation routines. It allows the user to create 3-D wireframe objects, to move them in conceptual space, and then to display projections of them on the video display.

Objects are created and moved about within a 3-D conceptual space. Following convention (Artwick, 1985; Hearn \& Baker, 1986), the coordinates of this conceptual space are referred to as world coordinates; the three world coordinates are represented here by single-precision numbers. Object points in world coordinates are projected onto a user-specified plane through an eyepoint; the standard technique for doing so is simple (see, e.g., Artwick, 1985; Hearn \& Baker, 1986). Both the projection plane and the eyepoint are defined by their world coordinates; in our case, the eyepoint is located at the origin. Scaling of the projected image size without change of perspective can be accomplished by varying the distance of the projection plane from the eyepoint. The final step in the display process is to map the projected image, expressed in projection plane coordinates, into the graphics screen image, expressed in screen coordinates. In the present case, the graphics screen is the medium-resolution graphics mode of the IBM color/graphics adapter.

One major advantage of using a 3-D to 2-D graphics program instead of ad hoc 2-D graphics programs is its generality. The researcher can create a wide variety of static and dynamic visual stimuli without having to develop special-purpose programs for each type of object (e.g., a cube or a sphere) or each type of motion (e.g., motion in depth or motion within a frontal plane). Provided that the program utilizes world coordinates for specifying the object and its motion, the user need only specify the object coordinates and the desired motion. With that done, the correct projective imagery simply falls out of program execution. Another advantage of this type of program is that the 3-D object and its time-varying projective imagery are already represented within the computer in a form that makes them readily accessible to a computer model of the process being investigated, such as Ullman's (1979) scheme for recovering 3-D structure from optical motion. Thus, the investigator obtains 
a much more intimate connection between theorizing and experimentation than is permitted with other experimental approaches.

\section{Program Description}

During the object editing stage, the user creates an object by defining the world coordinates of each of its vertices conceived as offsets relative to the center of the object. At this initial stage of editing, the object's center is located at the origin of space. As each vertex is defined, it is assigned a number. The user then creates a file that tells the editor which numbered vertices are to be connected. This line file, containing pairs of vertex numbers, specifies the lines of the object. The program allows for up to 32 vertices and 40 lines per object. The vertices file and the line file can be stored separately on disk. Because the line file contains only pairs of vertex numbers and no information about the coordinates of the vertices, it can be used to connect different sets of vertices in the same way. Alternatively, the same set of vertices can be connected in different ways with different line files. This feature might be useful for creating a number of similar objects varying slightly in shape.

The editor program creates objects using dotted lines with the dots arranged so that adjacent dots are equally spaced in world coordinates. This means that the dots generally are not equally spaced in the screen imagery. This can be seen in the two image sequences of Figure 1. (Dotted lines are used rather than solid lines to keep the number of illuminated pixels in each image frame within the limits required for high-speed animation using the routines presented earlier.) The program is set up to work with one to four objects; this default value, determined by the amount of memory available to BASICA, should be changed in the dimension statements if more objects of lesser complexity are desired.

Three kinds of rigid object motion can be simulated: rotation about an arbitrary fixed axis, linear translation in any direction, and any combination of the two. The motions of each object within a scene can be defined separately. A rotation is specified by an axis and the total rotation angle about that axis. Linear translations are specified by the world coordinates of the starting and ending locations.

In the mapping from projection plane to graphics screen, the user has control over the aspect ratio of the screen image. This makes it possible to take into account variations in the aspect ratios of various graphics adapters, video monitors, and printers. In addition, this mapping from projection plane to graphics screen permits control over the positioning of the imagery that is independent of that produced by the projection process; this is useful, for example, in creating stereoscopic displays in which the left and right eye views are positioned side by side on the screen.

Images can be displayed in any color supported by medium-resolution graphics. Objects in world coordinates are defined without regard to color; color is defined only a
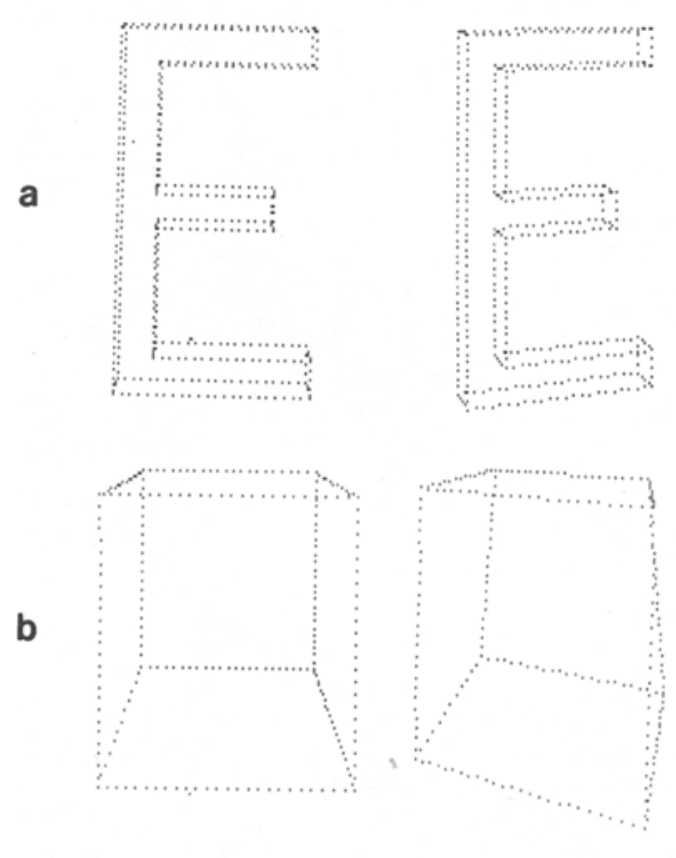
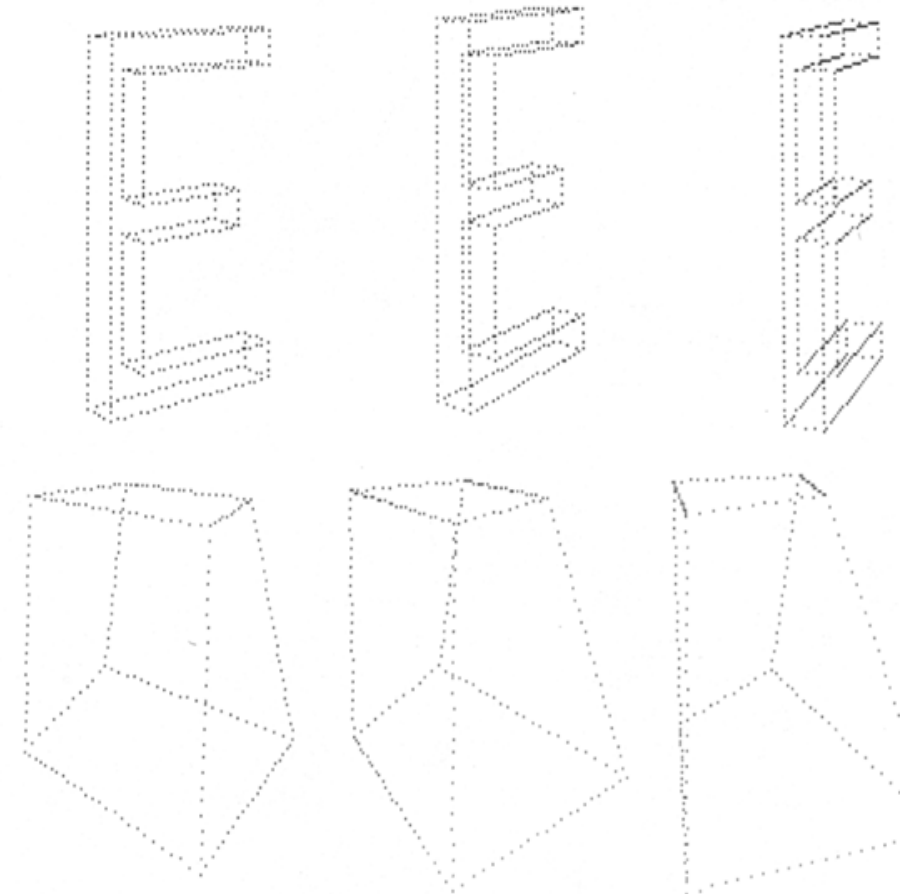

Figure 1. Five images from each of two animation sequences created using the $3-D$ graphics editor. (a) Rotation of the letter $\mathbf{E}$ about its vertical axis. (b) Rotation of an object that is projectively equivalent to a rectangular solid in its initial orientation. 
during the screen mapping. This means that the same object file can be used to create projective images of different colors.

The program is written so that a programmer can easily modify one section without interfering with the function of another. For example, we have replaced the wireframe object editor by editors for creating curved lines and textured surfaces, such as those used in studies of the perception of nonplanar surfaces (e.g., Braunstein, 1976; Todd, 1984; Uttal, 1985). Alternatively, the object editor can be modified with relative ease so that an object's shape or the elements defining its surface can be changed during successive frames of an animation sequence. The motion editor can be altered to produce more complex forms of movement, such as rotation of the axis of rotation (precession) or curvilinear translation. Finally, of course, routines can be added that perform functions such as hidden line elimination, object occlusion, or screen distortion.

\section{APPLICATIONS}

By themselves, the animation routines interfaced through BASIC or another high-level language lend themselves to many applications. These include study of the perception of motion in the frontal plane (Anstis, 1986; Badler \& Tsotsos, 1986; Hildreth, 1984; Mack, 1986), development of a multichannel tachistoscope, and display of time-series data. The graphics editor adds the capability of moving relatively complex objects in 3-D space, which includes the frontal plane. As such, it might find application in the study of the structure from motion problem (Badler \& Tsotsos, 1986; Braunstein, 1976; Todd, 1985; Ullman, 1979; Wallach \& O'Connell, 1953), the perception of motion in three dimensions (Badler \& Tsotsos, 1986; Regan, Kaufman, \& Lincoln, 1986), kinetic subjective contours (Bradley \& Lee, 1982), mental rotation of 3-D objects (Pinker \& Finke, 1980; Shepard \& Metzler, 1971), and the stereokinetic phenomenon (Regan et al., 1986).

\section{AVAILABILITY}

Two floppy disks are available from the authors. One contains the BASIC interface program (ANIMATE.BAS), the source and object files of the animation routines, and interpreter and compiler versions of the 3-D graphics editor. The other disk contains several animation sequences dealing with the perception of 3-D structure from optical motion (Ullman, 1979; Wallach \& O'Connell, 1953). Documentation of the graphics editor is also provided, including a brief discussion of 3-D to 2-D projective transformation and instructions on linking compiled programs with the animation object files. The floppy disks can be obtained by writing to either author. In order to cover the cost of the disks, duplication, and postage, please include a check made out to Regents of the University of California in the amount of $\$ 6$.

\section{REFERENCES}

ANSTIS, S. (1986). Motion perception in the frontal plane: Sensory aspects. In K. R. Boff, L. Kaufman, \& J. P. Thomas (Eds.), Handbook of perception and human performance: Volume 1. Sensory processes and perception (pp. 16.1-16.27). New York: Wiley.

ARTWICK, B. A. (1985). Microcomputer displays, graphics, and animation. Englewood Cliffs, NJ: Prentice-Hall.

BAdLer, N. I., \& Tsorsos, J. K. (Eds.). (1986). Motion: Representation and perception. New York: North-Holland.

BradLeY, D. R., \& LeE, K. (1982). Animated subjective contours: Perception \& Psychophysics, 32, 393-395.

BraUnSTEIN, M. L. (1976). Depth perception through motion. New York: Academic Press.

DAvies, R. (1985). COMPUTE!'s mapping the IBM PC and PCjr. Greensboro, NC: COMPUTE! Publications.

GIBson, J. J. (1950). The perception of the visual world. Boston: Houghton Mifflin.

GiBson, J. J. (1979). The ecological approach to visual perception. Boston: Houghton Mifflin.

HeARN, D., \& Baker, M. P. (1986). Computer graphics. Englewood Cliffs, NJ: Prentice-Hall.

HiLDRETH, E. C. (1984). The measurement of visual motion. Cambridge, MA: MIT Press.

MACK, A. (1986). Perceptual aspects of motion in the frontal plane. In K. R. Boff, L. Kaufman, \& J. P. Thomas (Eds.), Handbook of perception and human performance: Volume 1 . Sensory processes and perception (pp. 17.1-17.38). New York: Wiley.

Pinker, S., Finke, R. A. (1980). Emergent two-dimensional patterns in images rotated in depth. Journal of Experimental Psychology: Human Perception \& Performance, 6, 244-264.

Regan, D. M., Kaufman, L., \& Lincoln, J. (1986). Motion in depth and visual acceleration. In K. R. Boff, L. Kaufman, \& J. P. Thomas (Eds.), Handbook of perception and human performance: Volume 1. Sensory processes and perception (pp. 19.1-19.46). New York: Wiley.

Rollins, D. (1985). IBM PC 8088 Macro Assembler Programming. New York: Macmillan.

ShePARD, R. N., \& Metzler, J. (1971). Mental rotation of threedimensional objects. Science, 171, 701-703.

ToDD, J. (1984). The perception of three-dimensional structure from rigid and nonrigid motion. Perception \& Psychophysics, 36, 97-103.

ToDD, J. (1985). Perception of structure through motion: Is projective correspondence of moving elements a necessary condition? Journal of Experimental Psychology: Human Perception \& Performance, 11, 689-710.

Ullman, S. (1979). Interpretation of visual motion. Cambridge, MA: MIT Press.

UTTAL, W. R. (1985). The detection of nonplanar surfaces in visual space. Hillsdale, NJ: Erlbaum.

Wallach, H., \& O'ConNell, D. N. (1953). The kinetic depth effect. Journal of Experimental Psychology, 45, 205-217. 
LISTING 1

Source Code for ANIMATE.ASM

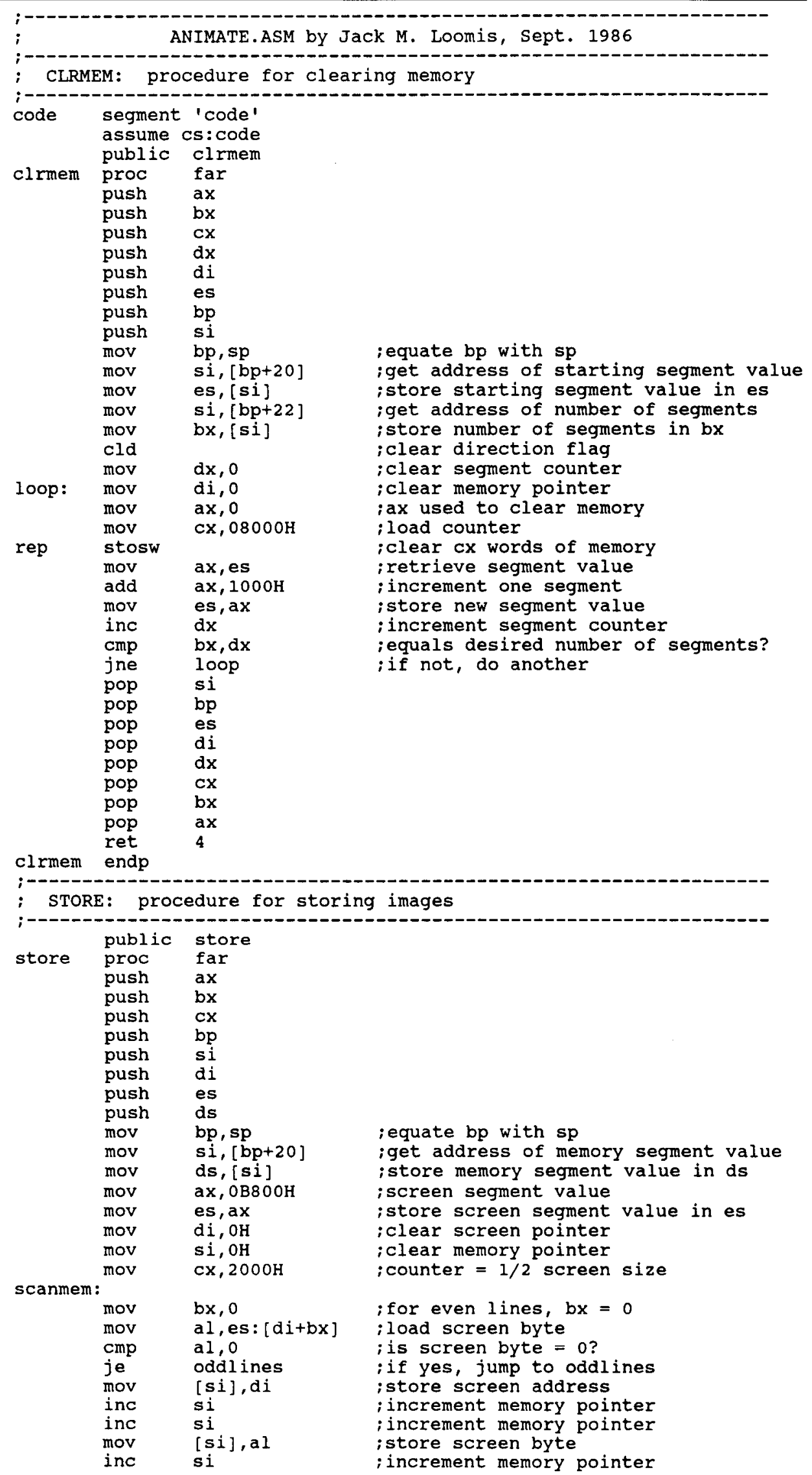


LISTING 1 (Continued)

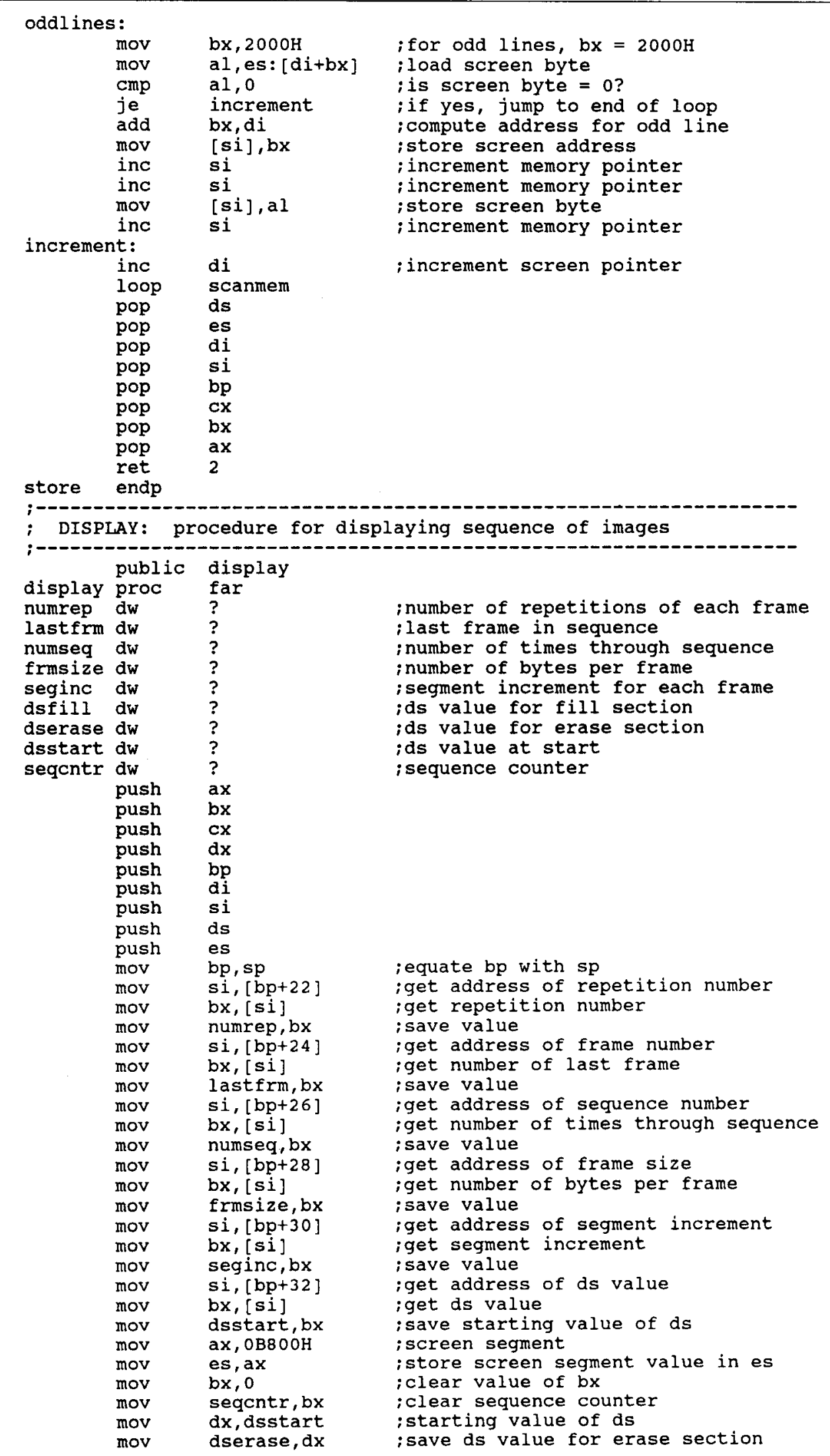


LISTING 1 (Continued)

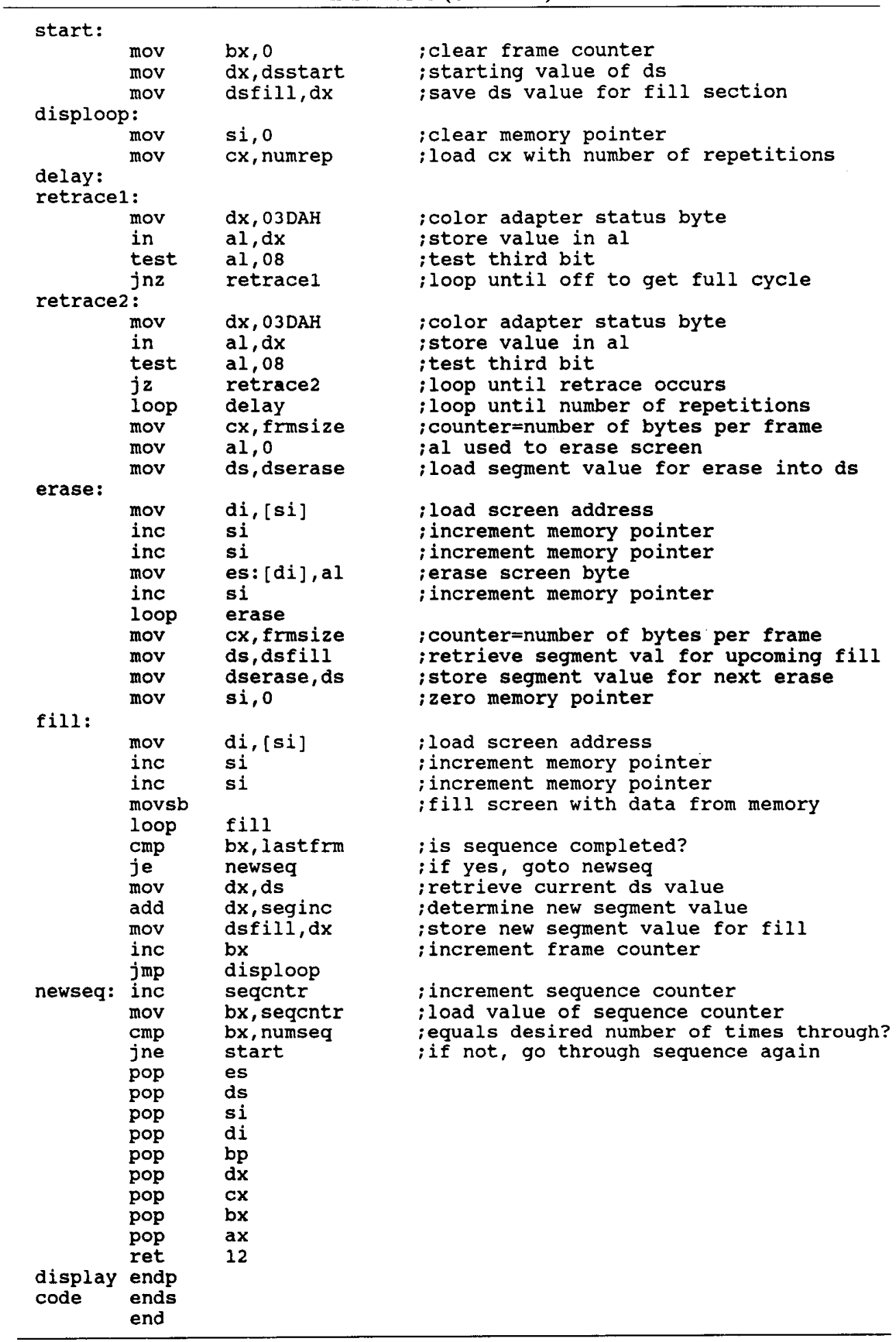


LISTING 2

BASIC Interface Program ANIMATE.BAS

\begin{tabular}{|c|c|}
\hline 10 & 'INITIALIZE \\
\hline 20 & CLS : KEY OFF : DEF SEG=\&H7F 94 \\
\hline 30 & FOR $I=0$ TO 385:READ V:POKE I,V:NEXT I \\
\hline 40 & CLRMEM $=\$ H O: S T O R E=\& H 3 A: D I S P L A Y=\& H 9 B$ \\
\hline 50 & INPUT "STARTING SEGMENT NUMBER $(2,3,4, \ldots)$ ";SN \\
\hline 60 & INPUT "NUMBER OF BYTES PER FRAME (MULTIPLE OF 16)";FRMSIZE\% \\
\hline 70 & INPUT "NUMBER OF FRAMES IN SEQUENCE"; NUMFRM : LASTFRM $\%=$ NUMFRM $\%-1$ \\
\hline 80 & NUMBYTES $=3 *$ FRMS I $2 E \% *$ NUMFRM $\%$ NUMBER $\%=$ INT $($ NUMBYTES $/ 65536 !)+1$ \\
\hline 90 & DSSTART $\%=4096 *$ SN $:$ SEGMENT $\%=$ DSSTART $\%$ \\
\hline 100 & SEGINC $\%=3 *$ FRMSIZE $\% / 16:$ 'SEGMENT INCREMENT \\
\hline 110 & CALL CLRMEM(NUMBER\%, DSSTART\%): 'CLEAR MEMORY \\
\hline 1201 & ' \\
\hline 130 & 'CREATE IMAGES AND STORE SEQUENTIALLY IN MEMORY \\
\hline 140 & SCREEN 1:COLOR 0,1:'MEDIUM RESOLUTION GRAPHICS \\
\hline 150 & FOR FRAME $=0$ TO LASTFRM\% \\
\hline 160 & CLS:'CLEAR VIDEO SCREEN PRIOR TO IMAGE CREATION \\
\hline 170 & GOSUB 2000:'CREATE IMAGE FOR PRESENT FRAME \\
\hline 180 & CALL STORE (SEGMENT\&) : 'STORE IMAGE IN MEMORY \\
\hline 190 & SEGMENT $\%=$ SEGMENT $\%+$ SEGINC $:$ NEXT FRAME \\
\hline 200 & 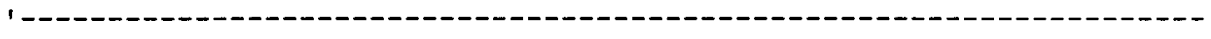 \\
\hline 210 & 'DISPLAY IMAGES FROM MEMORY IN RAPID SUCCESSION \\
\hline 220 & CLS: INPUT "NUMBER OF RASTER SCANS PER FRAME";NUMREP\% \\
\hline 230 & INPUT "NUMBER OF TIMES THROUGH SEQUENCE"; NUMSEQ\& \\
\hline 240 & CLS:CALL DISPLAY (DSSTART $\%$, SEGINC $\%$, FRMS IZE $\%$, NUMSEQ $\%$, LASTFRM $\%$, NUMREP \\
\hline 250 & CLS: PRINT "DO AGAIN? (Y/N)" \\
\hline 260 & RS=INKEY\$:IF R\$="" THEN 260 \\
\hline 270 & IF R\$="Y" THEN CLS:GOTO 220 \\
\hline 280 & SCREEN $0:$ WIDTH $80:$ END \\
\hline 1000 & 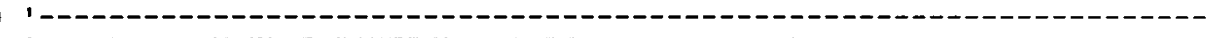 \\
\hline 1010 & 'MACHINE LANGUAGE ROUTINES STORED IN DATA STATEMENTS \\
\hline 1020 & DATA $80,83,81,82,87,6,85,86,139,236,139,118,20,142,4$ \\
\hline 1030 & DATA $139,118,22,139,28,252,186,0,0,191,0,0,184,0,0,185$ \\
\hline 1040 & DATA $0,128,243,171,140,192,5,0,16,142,192,66,59,218,117$ \\
\hline 1050 & DATA $233,94,93,7,95,90,89,91,88,202,4,0,80,83,81$ \\
\hline 1060 & DATA $85,86,87,6,30,139,236,139,118,20,142,28,184,0,184$ \\
\hline 1070 & DATA $142,192,191,0,0,190,0,0,185,0,32,187,0,0,38$ \\
\hline 1080 & DATA $138,1,60,0,116,7,137,60,70,70,136,4,70,187,0$ \\
\hline 1090 & DATA $32,38,138,1,60,0,116,9,3,223,137,28,70,70,136$ \\
\hline 1100 & DATA $4,70,71,226,217,31,7,95,94,93,89,91,88,202,2$ \\
\hline 1110 & DATA $0,0,0,0,0,0,0,0,0,0,0,0,0,0,0$ \\
\hline 1120 & DATA $0,0,0,0,80,83,81,82,85,87,86,30,6,139,236$ \\
\hline 1130 & DATA $139,118,22,139,28,46,137,30,136,0,139,118,24,139,28$ \\
\hline 1140 & DATA $46,137,30,138,0,139,118,26,139,28,46,137,30,140,0$ \\
\hline 1150 & DATA $139,118,28,139,28,46,137,30,142,0,139,118,30,139,28$ \\
\hline 1160 & DATA $46,137,30,144,0,139,118,32,139,28,46,137,30,150,0$ \\
\hline 1170 & DATA $184,0,184,142,192,187,0,0,46,137,30,152,0,46,139$ \\
\hline 1180 & DATA $22,150,0,46,137,22,148,0,187,0,0,46,139,22,150$ \\
\hline 1190 & DATA $0,46,137,22,146,0,190,0,0,46,139,14,136,0,186$ \\
\hline 1200 & DATA $218,3,236,168,8,117,248,186,218,3,236,168,8,116,248$ \\
\hline 1210 & DATA $226,238,46,139,14,142,0,176,0,46,142,30,148,0,139$ \\
\hline 1220 & DATA $60,70,70,38,136,5,70,226,246,46,139,14,142,0,46$ \\
\hline 1230 & DATA $142,30,146,0,46,140,30,148,0,190,0,0,139,60,70$ \\
\hline 1240 & DATA $70,164,226,249,46,59,30,138,0,116,15,140,218,46,3$ \\
\hline 1250 & DATA $22,144,0,46,137,22,146,0,67,235,161,46,255,6,152$ \\
\hline 1260 & DATA $0,46,139,30,152,0,46,59,30,140,0,117,131,7,31$ \\
\hline 1270 & DATA $94,95,93,90,89,91,88,202,12,0$ \\
\hline 2000 & ' \\
\hline 2010 & 'CREATE IMAGE FOR PRESENT FRAME \\
\hline 2020 & $X=2 \star F R A M E: ' X$ COORDINATE OF LEFT EDGE OF RECTANGLE \\
\hline 2030 & $\operatorname{LINE}(X, 140)-(X+25,140): \operatorname{LINE}(X+25,140)-(X+25,180)$ \\
\hline 2040 & $\operatorname{LINE}(X+25,180)-(X, 180): \operatorname{LINE}(X, 180)-(X, 140):$ RETURN \\
\hline
\end{tabular}

(Manuscript received July 8, 1986;

revision accepted for publication December 12,1986 .) 